\title{
L'INFLUENCE DE LA DURÉE DES INTERVALLES DES TRAITES SUR LA SÉCRÉTION DU LAIT ET SA TENEUR EN MATIËE GRASSE
}

\author{
par l'Ingénieur GROH
}

Chef de la Section de Lactologie à l'Institut Provincial de Recherches Zootechniques à Brno, Cerna Pole (Tchécoslovaquie).

Il y a beaucoup d'auteurs qui se sont occupés de la question de savoir combien de fois on devait traire, et qui ont appliqué leur attention spécialement à rechercher s'il était plus avantageux de traire deux fois ou trois fois par jour. L'objet du présent travail était d'établir plus précisément les relations existant entre la durée des intervalles entre les traites et la quantité ainsi que la teneur en matière grasse du lait produit par la race hanaque-bernoise, dont les animaux à rapport combiné sont élevés dans quelques contrées de la République Tehécoslovaque.

Les deux premières expériences, faites à titre d'information, furent exécutées à l'étable de l'Institut avec 3 laitières hanaquebernoises, dont l'aptitude productrice avait été déjà examinée. Leur poids vif ne changeait au cours de l'expérience que d'une façon infime. La ration quotidienne était proportionnée au rendement laitier des vaches et ne changeait pas pendant l'expérience. La durée des périodes partieulières était, dans les deux expériences, de trois jours; quoique courte, elle suffit, ear les conditions des trois vaches étaient, pendant tous les jours d'observation, à peu près égales. Les vaches furent affourragées toujours au moment de la traite pendant les périodes au cours desquelles les intervalles entre les traites furent raccourcis et la ration quotidienne de fourrage fut toujours divisée en lots tels qu'ils correspondaient approximativement à la durée du prochain intervalle.

La première expérience fut effectuée du 13 février au 24 mars 1928 et fut divisée en 13 périodes. Le moment de la traite fut changé pendant les périodes particulières comme il est indiqué dans le tableau I.

Les changements de l'heure de traite n'avaient aucune influence perturbatrice sur la production laitière et non plus sur la production de la matière grasse, car le rendement laitier s'accrut au cours de l'expérience de $0 \mathrm{~kg}$. 254 et la quantité de matière grasse augmenta de 45 gr. 2 pendant la dernière période par rapport à la première. Seulement au cours de la septième et neuvième période fut gagnée une quantité de lait inférieure à celle qui correspond à la ligne de la moyenne de la première et treizième périodes. Les oscillations de la quantité de lait n'étaient pas grandes au cours de l'expérience et la plus grande différence se produisait à la troisième période, en faisant 
TABLEAU I.

\begin{tabular}{|c|c|c|c|c|c|c|c|c|c|}
\hline \multirow{2}{*}{$\begin{array}{l}\text { Période } \\
\text { I } \ldots \ldots \ldots\end{array}$} & \multicolumn{4}{|c|}{ Heure de la traite } & \multirow{2}{*}{$\frac{\text { Période }}{\text { VIII } \ldots \ldots}$} & \multicolumn{4}{|c|}{ Heure de la traite } \\
\hline & 6 & 12 & 18 & - & & 6 & 12 & - & 24 \\
\hline II & 8 & 12 & 20 & - & IX .... & - & 12 & 18 & 24 \\
\hline III & 10 & 12 & 22 & - & $\mathrm{x} \ldots \ldots$ & 6 & T & 18 & 24 \\
\hline IV .... & - & 12 & - & 24 & $\mathrm{XI}$. & 6 & 12 & 18 & 24 \\
\hline V... & 4 & 12 & 16 & - & $\mathrm{XII}$ & 4 & 12 & 20 & - \\
\hline VI & 2 & 12 & 14 & - & XIII & 6 & 12 & 18 & - \\
\hline VII. & 6 & - & 18 & - & & & & & \\
\hline
\end{tabular}

RÉSULTATS DE LA PREMIÈRE EXPÉRIENCE.

Ces résultats sont rendus évidents par le tableau II et le graphique $n^{0} 1$.

\section{GRAPHIQUE N:T}

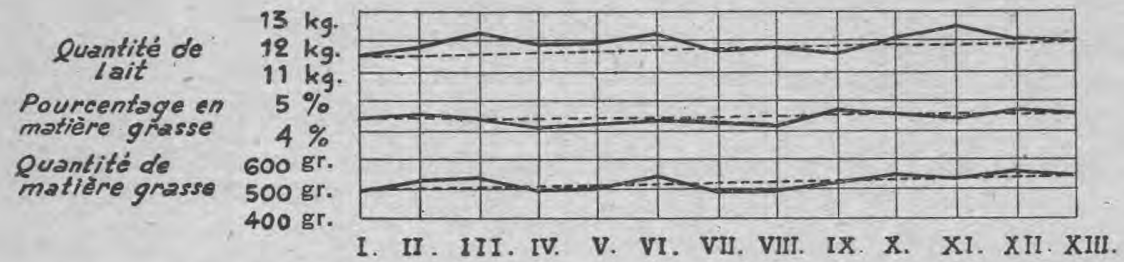

$0 \mathrm{~kg}$. 883. Egalement, les changements dans le pourcentage de matière grasse n'étaient pas forts et la plus grande différence à la douzième et huitième périodes s'élevait à $0,5 \%$.

Pendant les trois premières périodes, on suivit dans la traite triple, l'influence du raccourcissement de l'intervalle du matin et de la prolongation de celui de l'après-midi sur la quantité et le pourcentage de matière grasse du lait. La quantité de lait et de matière grasse obtenue par jour augmenta successivement, le pourcentage de matière grasse s'accrut pendant la deuxième période par rapport à la première, mais, pendant la troisième, il se maintint à la hauteur de la deuxième période. Au raccourcissement des intervalles matinaux de 6 à 4 et à 2 heures, qui suivirent après les intervalles de durée égale de 12 heures, la quantité absolue du lait de midi diminua, il est vrai, mais l'intensité de la sécrétion (quantité par heure) 
TABLEAU II.

\begin{tabular}{|c|c|c|c|c|c|c|c|c|c|c|c|c|c|c|c|}
\hline \multirow[b]{2}{*}{ Période } & \multicolumn{5}{|c|}{$\begin{array}{c}\text { Quantité moyenne de lait } \\
\text { (en kilogrammes) }\end{array}$} & \multicolumn{5}{|c|}{$\begin{array}{c}\text { Pourcentage moyen de matière } \\
\text { grasse }\end{array}$} & \multicolumn{5}{|c|}{$\begin{array}{l}\text { Quantité moyenne de matière grasse } \\
\text { (en grammes) }\end{array}$} \\
\hline & Matin & Midi & Soir & Minuit & $\begin{array}{c}\text { Total } \\
\text { par } \\
\text { jour }\end{array}$ & $\begin{array}{l}\mathrm{Ma}- \\
\text { tin }\end{array}$ & Midi & Soir & $\begin{array}{l}\text { Mi- } \\
\text { nuit }\end{array}$ & $\begin{array}{c}\text { Moyenne } \\
\text { par } \\
\text { jour }\end{array}$ & Matin & Midi & Soir & Minuit & $\begin{array}{c}\text { Total } \\
\text { par } \\
\text { jour }\end{array}$ \\
\hline $\mathbf{I} \ldots \ldots \ldots \ldots \ldots \ldots \ldots \ldots$ & 5,563 & 3,242 & 2,753 & - & 11,558 & 3,13 & 5,87 & 4,60 & - & 4,25 & 174,1 & 190,3 & 126,7 & 一 & 491,1 \\
\hline II $\ldots \ldots \ldots \ldots \ldots \ldots$ & 5,573 & 2,300 & 4,043 & - & 11,916 & 3,59 & 6,65 & 4,25 & - & 4,40 & 199,9 & 153,0 & 172,0 & - & 524,9 \\
\hline III $\ldots \ldots \ldots \ldots \ldots \ldots \ldots \ldots$ & 5,855 & 1,413 & 5,099 & - & 12,367 & 3,94 & 7,21 & 4,10 & - & 4,38 & 230,6 & 101,9 & 208,9 & - & 541,4 \\
\hline IV $\ldots \ldots$, & - & 5,883 & - & 5,941 & 11,824 & - & 4,56 & - & 3,70 & 4,13 & - & 268,4 & - & 220,0 & 488,4 \\
\hline $\mathrm{V} \ldots \ldots \ldots \ldots \ldots \ldots$ & 5,587 & 4,190 & 2,144 & - & 11,921 & 3,09 & 5,00 & 5,85 & - & 4,26 & 172,4 & 209,6 & 125,5 & - & 507,5 \\
\hline VI $\ldots \ldots \ldots \ldots \ldots \ldots \ldots \ldots$ & 5,593 & 5,333 & 1,292 & - & $12,21 \mathrm{~s}$ & 3,49 & 4,44 & 7,99 & - & 4,38 & 195,3 & 236,9 & 103,2 & - & 535,4 \\
\hline VII $\ldots$. & 5,566 & - & 5,918 & - & 11,484 & 3,77 & 一 & 4,60 & - & 4,20 & 209,8 & - & 272,0 & - & 481,8 \\
\hline VIII $\ldots \ldots$ & 3,154 & 3,073 & - & 5,655 & 11,882 & 4,97 & 4,79 & - & 3,29 & 4,12 & 156,7 & 147,2 & - & 185,8 & 489,7 \\
\hline IX $\ldots \ldots$ & - & 5,669 & 2,992 & 2,843 & 11,504 & - & 4,17 & 5,42 & 4,56 & 4,59 & - & 236,3 & 162,3 & 129,6 & 528,2 \\
\hline $\mathrm{X} \ldots \ldots$ & 3,104 & - & 5,731 & 3,263 & 12,098 & 5,00 & - & 3,67 & 5,69 & 4,56 & 155,2 & 一 & 210,3 & 185,6 & 551,1 \\
\hline XI & 3,204 & 2,996 & 3,006 & 3,106 & 12,312 & 4,44 & 4,63 & 3,90 & 4,14 & 4,28 & 142,3 & 138,6 & 117,3 & 128,5 & 526,7 \\
\hline XII . . . & 4,031 & 3,964 & 4,068 & + & 12,063 & 4,64 & 4,51 & 4,71 & - & 4,62 & 187,0 & 178,6 & 191,5 & - & 557,1 \\
\hline XIII $\ldots \ldots \ldots \ldots \ldots$ & 5,579 & 3,299 & 2,934 & - & 11,812 & 3,49 & 5,87 & 5,04 & 一 & 4,54 & 194,5 & 193,8 & 148,0 & - & 536,3 \\
\hline
\end{tabular}


augmenta de 540,3 à 575 et à 706 gr. 5, et elle fut, en ce qui concerne la moyenne quotidienne, de 58,7, 78,5 et de 191 gr. 2 plus grande. De même, la quantité de la matière grasse obtenue diminua, mais l'intensité de sa formation augmenta de 31,7 à 38,3 et à 51 grammes par litre, et, dans sa différence par comparaison à la moyenne quotidienne, elle fut de $11,2,16,4$ et 28 gr. 4 plus grande. Le pourcentage de matière grasse accusa un accroissement subit de 5,87 à 6,65 et à 7,21 et il fut, par rapport à la moyenne quotidienne, de $1,62,2,25$ et 2,83 plus grand.

A la traite matinale, après des intervalles de durée égale, auxquels précédèrent des intervalles d'après-midi progressivement prolongés, un lait fut obtenu dont le pourcentage s'acerut de 3,13 à 3,59 et à 3,94 et la différence s'affaiblit, par rapport à la moyenne quotidienne, de $-1,12$ à $-0,81$ et à $-0,44 \%$. L'intensité de la sécrétion de la matière grasse augmenta de 14,5 à 16,7 et à $19 \mathrm{gr}, 2$ et la différence diminua, par rapport à la moyenne quotidienne, de $-6,0$ à $-5,2$ et à $-3 \mathrm{gr}$. 4. La prolongation progressive des intervalles d'après-midi qui suivirent aux intervalles décroissants d'avant-midi eut pour suite une diminution du pourcentage gras du lait du soir de 4,60 à 4,25 et à 4,10 , alors que la différence soustraite de la moyenne quotidienne fut, pendant la première période, encore positive en comportant $0,35 \%$, tandis que, pendant la deuxième et troisième période, elle fut négative et comportait $-0,15$, e'est-à-dire $-0,28 \%$. La quantité absolue de lait augmenta, mais l'intensité de sa formation diminua (à l'exception de la deuxième période pendant laquelle également l'intensité de la sécrétion laitière accusa une déviation soustraite à la moyenne quotidienne), alors que la différence soustraite à la moyenne quotidienne fut pendant la première période encore positive en comportant 0 gr. 6 , tandis que pendant les deux périodes suivantes elle fut négative et comportait $-0,4$ et $-1 \mathrm{gr}$. 7 .

Pendant la quatrième période, les traites furent faites à des intervalles égaux à midi et à minuit et la quantité de lait produit fut à peu près égale, mais le pourcentage gras du lait obtenu après la deuxième moitié du jour fut plus bas.

Pendant la cinquième et la sixième période, les intervalles d'aprèsmidi entre les traites furent raccourcis et ceux du matin furent prolongés, en laissant les intervalles de nuit inchangés. Pendant ces périodes, des conditions similaires à celles des trois premières périodes furent déterminées.

Pendant la septième période, les traites furent faites de nouveau après des intervalles de matin et de soir égaux. La quantité de lait et de matière grasse produite au cours de la moitié du jour fut plus grande et son pourcentage gras plus haut que celle qui fut produite pendant la moitié de nuit. 
Pendant la huitième, la neuvième et la dixième période, auxquelles s'adjoignirent aussi les première et treizième périodes, les traites furent faites de manière à alterner par intervalles de 6 heures avec ceux de 12 heures dans les différentes parties de la journée. Comme résultat, on établit que, pendant les intervalles plus courts qui suivirent les intervalles plus longs, l'intensité de la formation du lait et de la matière grasse augmentait considérablement et que le pourcentage gras s'accroissait. Pendant les intervalles auxquels précédèrent les intervalles de durée éagle, l'intensité de la formation du lait diminuait par rapport au premier cas, l'intensité de la formation de la matière grasse et le pourcentage gras diminuaient, mais dans tous les cas ils étaient plus forts que pendant les intervalles plus longs.

Pendant la onzième et la douzième période, les changements dans la production du lait et de la matière grasse furent poursuivis à des intervalles de durée égale entre les traites. La quantité du lait et son contenu absolu et relatif en matière grasse accusaient, autant aux intervalles de 6 heures qu'à ceux de 8 heures, des oscillations.

La deuxième expérience fut effectuée du 16 au 30 avril 1929, en 5 périodes, dont les heures de traite sont évidentes d'après le tableau III. Les résultats de cette expérience figurent dans les tableaux III et IV et dans le graphique $n^{\circ} 2$.

La production quotidienne du lait s'acerut au cours de l'expérience, tandis que le pourcentage gras resta presque inchangé. De ce fait, on peut juger que les changements de l'heure de la traite n'avaient pas une influence défavorable sur la sécrétion lactée.

La quantité de la production quotidienne du lait s'accrut pendant la deuxième période, où les traites furent faites six fois par jour à

TABLEAU III.

QUANTITÉ MOYENNE DE LAIT (kilogrammes).

\begin{tabular}{|c|c|c|c|c|c|c|c|c|c|c|c|c|c|}
\hline \multirow{2}{*}{$\begin{array}{c}\text { Pério- } \\
\text { de: }\end{array}$} & \multicolumn{12}{|c|}{ Heure de la traite } & \multirow{2}{*}{$\begin{array}{c}\text { Total } \\
\text { par } \\
\text { jour }\end{array}$} \\
\hline & 6 & 9 & 10 & 12 & 14 & 15 & 18 & 21 & 22 & 24 & 2 & 3 & \\
\hline I $\ldots$ & 4,267 & - & - & 2,196 & - & - & 2,111 & - & - & 一 & - & 一 & 8,574 \\
\hline II & 1,364 & - & 1,387 & - & 1,725 & - & 1,602 & - & 1,534 & - & 1,690 & - & 9,302 \\
\hline III & 4,622 & - & - & - & - & - & 4,302 & - & - & - & - & - & 8,924 \\
\hline IV .. & 1,271 & 1,124 & - & 1,049 & - & 1,300 & 1,233 & 1,049 & 一 & 1,370 & - & 0,777 & 9,173 \\
\hline V & 4,796 & - & - & 2,267 & - & - & 2,105 & - & - & - & - & - & 9,168 \\
\hline
\end{tabular}


TABLEAU IV.

POURCENTAGE MOYEN DE MATIÈRE GRASST,

\begin{tabular}{|c|c|c|c|c|c|c|c|c|c|c|c|c|c|}
\hline \multirow{2}{*}{ Période : } & \multicolumn{12}{|c|}{ 1" Heure de la traite } & \multirow{2}{*}{\begin{tabular}{|c} 
Nojann \\
par \\
jour
\end{tabular}} \\
\hline & 6 & 9 & 10 & 12 & 14 & 15 & 18 & 21 & 22 & 24 & 2 & 3 & \\
\hline I & 3,73 & - & - & 4,95 & - & - & 4,24 & - & - & 一 & - & - & 4,17 \\
\hline II $\ldots \ldots$. & 4,06 & - & 3,69 & - & 3,66 & - & 4,28 & - & 3,80 & - & 3,98 & - & 3,91 \\
\hline III $\ldots \ldots \ldots$. & 4,00 & - & - & - & - & - & 4,33 & - & - & - & - & - & 4,16 \\
\hline IV. & 4,67 & 4,35 & - & 3,98 & - & 3,95 & 4,40 & 3,76 & - & 4,36 & - & 3,60 & 4,17 \\
\hline V & 3,63 & - & - & 5,09 & - & 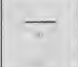 & 4,23 & - & - & - & - & - & 4,13 \\
\hline
\end{tabular}

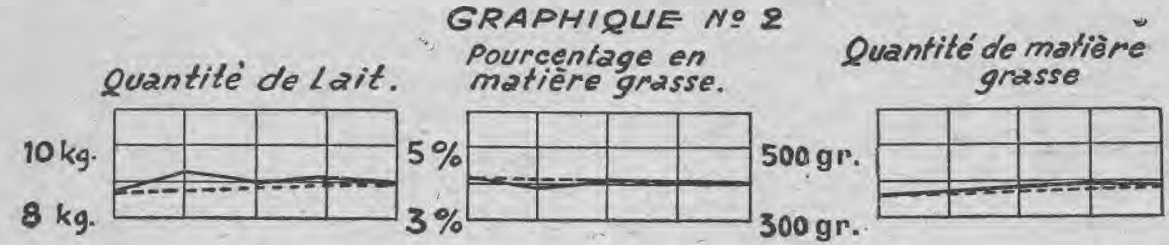

intervalles de 4 heures, sensiblement au-dessus de la moyenne de la première et cinquième périodes, où les traites furent faites normalement trois fois par jour; d'autre part, le pourcentage gras s'abaissa pendant la deuxième période, mais la quantité absolue de matière grasse resta tout de même au-dęssus de la ligne de moyenne de la première et cinquième période.

Pendant la troisième période, où les traites furent faites deux fois par jour, la quantité de lait quotidienne diminua par rapport à la deuxième période, mais elle resta an-dessus de la moyenne de la première et cinquième période; par contre, pendant la troisième période, le poureentage et le contenu absolu en matière grasse augmentèrent.

Pendant la quatrième période, où les traites furent faites huit fois par jour à intervalles de 3 heures, la quantité de lait s'accrut de nouveau et le poureentage gras resta au niveau de celui de la période précédente, de sorte que la quantité absolue de la matière grasse atteignit le maximum de toutes les périodes. Quoique les intervalles entre les traites fussent égaux pendant la deuxième et la quatrième période ( 4 et 3 heures), la quantité et la teneur en matière grasse du lait recusilli après les intervalles particuliers accusaient cependant des oscillations considérables, surtout pendant la qua- 
trième période, sans que la quantité du lait ait fait voir pendant les intervalles particuliers une relation quelconque avec son pourcentage de matière grasse.

Deux autres expériences furent effectuées à la ferme expérimentale de Branky, dont la première fut poursuivie du 15 au 31 juillet 1928, à l'époque de l'affourragement estival et de pâturage, tandis que la deuxième fut faite à l'époque de l'affourragement hivernal du 7 au 23 avril 1929. Pour la première, on utilisa 10 laitières, et pour la deuxième, 9 laitières de la race hanaque-bernoise. La ration fourragère quotidienne administrée en 3 parties correspondait au rendement laitier et ne fut pas changé pendant toute l'expérience. Chacune des deux expériences fut divisée en 4 périodes de 4 jours. Pendant la première et la dernière période de ces deux expériences, la traite fut faite à 6,12 et 18 heures; pendant la deuxième période, à 4,12 et 20 heures, et pendant la troisième, à 6 et 18 heures. Le lait de chaque vache fut pesé à chaque traite et son contenu en matière grasse fut estimé. Des résultats ainsi obtenus on a ensuite tiré les moyennes quotidiennes.

\section{RÉSUltats DES EXPÉRIENCES.}

Ces résultats sont indiqués dans le tableau $\mathrm{V}$ et dans les graphiques nos 3 et 4 .

Au cours de la première expérience, le rendement laitier diminua de $0 \mathrm{~kg} .90$, et, au cours de la deuxième, de $0 \mathrm{~kg}$. 41 . Dans chacune des deux expériences, une diminution du rendement du lait apparut pendant la deuxième période par rapport à la première, mais la quantité de lait resta pourtant au-dessus de la moyenne de la première et de la quatrième période ; pendant la troisième période, elle tomba fortement au-dessous de la moyenne de la première et de la quatrième période. De même, le pourcentage gras diminua au cours des expériences : dans la première, de $0,15 \%$, mais au deuxième cas seulement de $0,03 \%$. Pendant la deuxième période de chacune des deux expériences le pourcentage gras décrut au minimum; pendant la troisième, il augmenta, il est vrai, par rapport à la deuxième, mais il n'atteignit pas la moyenne de la première et de la quatrième période. La plus petite quantité de matière grasse fut obtenue pendant la troisième période ; cependant, la moyenne de la première et de la quatrième ne fut pas atteinte non plus pendant la deuxième période. En examinant la première et la quatrième périodes, on trouve les mêmes relations entre la durée des intervalles d'une traite à l'autre et la quantité du lait, son pourcentage gras et la quantité absolue de la matière grasse, comme pendant les périodes analogues de l'expérience décrite au début. Seulement, les différences ne sont pas si marquées, et, pendant la quatrième, période de la première expé- 
TABLEAU V.

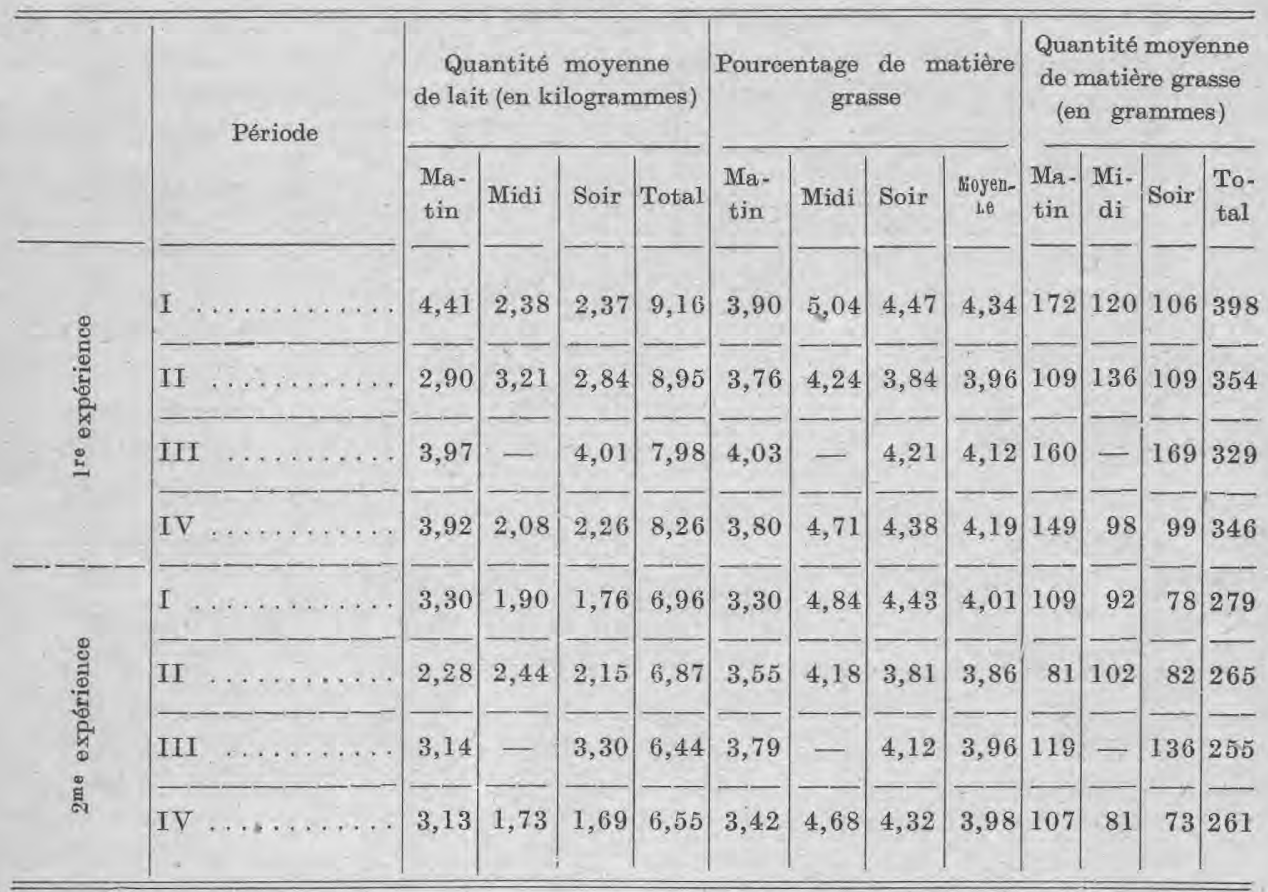

Expérience $I$

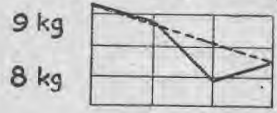

GRAPHIQUES HO 3 et 4
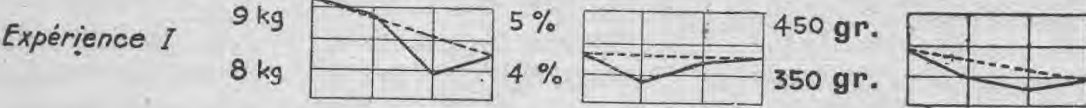

Quandité de Lait

Pourcentage en

Quantité de matière grasse

\section{Expérience 2}
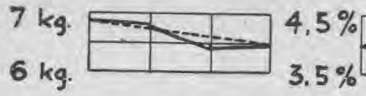

$300 \mathrm{gr}$.
$200 \mathrm{gr}$.

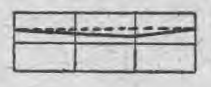

rience, une exception se manifeste dans la quantité du lait et dans l'intensité de la formation de la matière grasse. La quantité de lait produite pendant la moitié diurne de la journée surpasse, à la double traite dans la première expérience, celle qui fut produite pendant la moitié nocturne de la journée de 3,7 et de 5,08\% et, dans la deuxième expérience, cette différence fait 5,18 et $4,42 \%$. Pendant la moitié diurne on obtint, dans la première expérience, de 13,56 et de $13,88 \%$ et, dans la deuxième, de 21,86 et de $18 \%$ plus de matière grasse, qu'à la traite du matin après la moitié nocturne. Pendant la deuxième période, des deux expériences, la plus grande quantité de lait fut obtenue à la traite de midi; on en obtint moins à celle du matin et encore moins à celle du soir. Le lait de midi était le plus gras, ensuite 
celui dusoir et, en dernier lieu, celui du matin. La plus grande quantité de matière grasse fut obtenue dans le lait de midi, tandis que ceux du matin et du soir en contenaient des quantités égales. Pendant la troisième période de la première expérience, la production du lait était, au cours de l'intervalle diurne, de $0,5 \%$ et celle de la matière grasse de $2,74 \%$ plus grande qu'au cours de la nuit; dans la deuxième expérience, cette différence faisait 2,48 pour le lait et $6,66 \%$ pour la matière grasse.

Dans le but d'établir la différence apparaissant entre la double et la triple traite sur la production du lait et sa teneur en matière grasse, qui s'installe dans les conditions économiques de la circonscription de l'élevage hanaque-bernois, une expérience fut faite à Branky, du 17 février au 27 mars 1932, avec 15 laitières de cette race. Elle fut divisée en 4 périodes, chacune de 2 journées préparatoires et 8 journées expérimentales. La ration fourragère correspondait au rendement laitier des animaux et elle ne fut pas changée pendant toute l'expérience. Pendant la première, la deuxième et la quatrième période, on administra la ration quotidienne trois fois, en tiers, par jour, tandis que, pendant la troisième, on administra le matin et à midi toutes les fois un quart et le soir une moitié. La traite fut faite pendant les première et quatrième périodes à 6,12 et 18 heures, et, pendant la deuxième et la troisième, à 6 et 18 heures.

Les résultats de cette expérience sont évidents d'après le tableau VI et le graphique $n^{0} 5$.

TABLEAU VI.

\begin{tabular}{|c|c|c|c|c|c|c|c|c|c|c|c|c|}
\hline \multirow{2}{*}{ Période: } & \multicolumn{4}{|c|}{$\begin{array}{l}\text { Quantité moyenne de lait } \\
\text { (en kilogrammes) }\end{array}$} & \multicolumn{4}{|c|}{$\begin{array}{l}\text { Pourcentage moyen } \\
\text { de matière grasse }\end{array}$} & \multicolumn{4}{|c|}{$\begin{array}{c}\text { Quantité moyenne de matière } \\
\text { grasse (en grammes) }\end{array}$} \\
\hline & $\begin{array}{l}\mathrm{Ma} \\
\text { tin }\end{array}$ & Midi & Soir & Total & $\begin{array}{l}\mathrm{Ma}- \\
\mathrm{tin}\end{array}$ & Midi & Soir & $\begin{array}{l}\text { Moyen- } \\
\text { ne }\end{array}$ & matin & Midi & Soir & Total \\
\hline$I \ldots \ldots \ldots$ & 4,97 & 2,70 & 2,53 & 10,20 & 3,94 & 5,26 & 4,92 & 4,53 & 195,90 & 142,01 & 124,41 & 462,32 \\
\hline II $\ldots$. & 4,95 & - & 4,95 & 9,90 & 4,39 & - & 4,50 & 4,44 & 217,12 & - & 222,71 & 439,83 \\
\hline III $\ldots$. & 4,68 & - & 4,82 & 9,50 & 4,44 & - - & 4,36 & 4,40 & 207,62 & - & 210,04 & 417,66 \\
\hline IV $\ldots \ldots \ldots$ & 4,81 & 2,53 & 2,47 & 9,81 & 3,91 & 5,09 & 4,75 & 4,42 & 188,10 & 128,66 & 117,32 & 434,08 \\
\hline
\end{tabular}

Le rendement laitier diminua au cours de l'expérience au fur et à mesure que la lactation avança, de $0 \mathrm{~kg}$. 39. Le pourcentage en matière grasse décrut de $0,11 \%$.

Pendant la première période, on obtint, avec deux traites au 


\section{GRAPHIQUE N:5}

\section{Quantité de lait}

\section{Pourcentage en} matiére grasse.
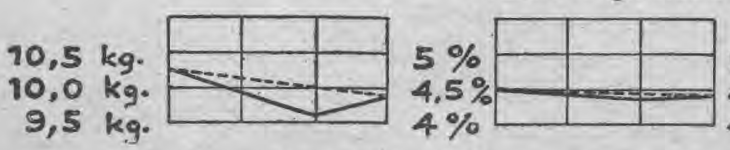

500. $\mathrm{gr}$. $450 \mathrm{gr}$. $400 \mathrm{gr}$.

ntité de matière grasse

cours de la moitié diurne, 2,54\% plús de lait et $15,26 \%$ plus de matière grasse qu'après la moitié nocturne avec une traite ; pendant la quatrième période, cette différence atteignait 1,94\% pour le lait et $13,34 \%$ pour la matière grasse. Pendant la deuxième période, la quantité de lait obtenue le matin fut égale à celle du soir, mais le dernier fut de $0,11 \%$ plus gras, ce qui permit d'obtenir le soir 5 gr. 59 , soit $1,28 \%$ plus de matière grasse que le matin. Pendant la troisième période, on obtint le soir $0 \mathrm{~kg} .14(1,48 \%)$ de lait de plus que le matin, mais son pourcentage gras fut de $0,08 \%$ plus bas, ce qui fit que le lait du soir ne contenait que $2 \mathrm{gr}, 42(0,58 \%)$ de plus de matière grasse par rapport à celui du matin. Pendant la deuxième période, on obtint $0 \mathrm{~kg}$. $17(1,69 \%)$ de lait en moins et $13 \mathrm{gr}$. 08 $(2,89 \%)$ de matière grasse en moins; pendant la troisième période, 0 kg. $44(4,43 \%)$ de lait en moins et 25 gr. $83(5,82 \%)$ de matière grasse en moins par comparaison aux moyennes respectives de la première et de la quatrième périodes.

\section{Conclusion.}

Les études effectuées avec les vaches laitières de la race hanaquebernoise ont montré que :

Si l'on trait trois fois par jour, le changement de l'heure de la traite ne provoque que des différences insensibles dans la quantité et dans le pourcentage en matière grasse du lait produit quotidiennement. Les changements de l'heure de la traite n'influent pas de manière troublante sur la sécrétion lactée, si les autres circonstances ne ehangent pas.

L'intensité de la formation du lait et de la matière grasse, de même que le pourcentage du lait en matière grasse, dépendent, non seulement de la durée de l'intervalle précédant la dernière traite, mais aussi de la durée du temps s'écoulant avant la traite précédente.

Si à des intervalles plus longs, mais de durée égale, ou à des intervalles de durée croissante, précédant la traite, suivent des intervalles courts, l'intensité de la formation du lait et de la matière grasse augmente, le pourcentage du lait en matière grasse s'accroît, et ceci d'autant plus que les intervalles sont plus courts et que les intervalles précédant ces intervalles courts sont plus longs. La prolongation 
croissante des intervalles a pour effet une intensité décroissante de la formation de la matière grasse ainsi que du pourcentage du lait en matière grasse, fait auquel s'ajoute aussi l'influence de la durée du temps s'écoulant avant la traite précédente. La quantité de lait et son pourcentage en matière grasse dépendent de même, pour des intervalles égaux entre les traites, de la durée des intervalles précédents. Si la traite est faite trois fois par jour, une fois après un intervalle plus long, et deux fois après des intervalles plus courts, mais de durée égale, on obtient après l'intervalle plus court qui suit l'intervalle plus long, plus de lait et un lait plus gras qu'après l'intervalle plus court auquel précédait un intervalle de durée égale. Le pourcentage en matière grassse plus fort du lait obtenu à une certaine heure diurne pendant des traites plus nombreuses, va au préjudice du pourcentage en matière grasse du lait obtenu après l'intervalle plus long non interrompu par la traite.

Si l'on trait plus de trois fois par jour, on obtient chez les vaches qui produisent 9 kilogrammes de lait environ, plus de lait, il est vrai, mais la différence est très faible et le pourcentage en matière grasse se tient dans les limites des oscillations quotidiennes. On ne peut alors influer sur le pourcentage en matière grasse du lait par des traites plus nombreuses que dans une mesure restreinte. Si l'on trait deux fois par jour les vaches qui donnent 10 kilogrammes de lait environ, on obtient pendant l'affourragement hivernal, en administrant la ration fourragère normale à l'heure d'affourragement normale, $2 \%$ environ moins de lait et $3 \%$ environ moins de matière grasse que si l'on trait trois fois par jour.

\title{
ANALYSE BACTÉRIOLOGIQUE DES BEURRES DANS LA RÉGION DE BREST. BIOLOGIE DU BACILLE TYPHIQUE DANS LE BEURRE
}

\author{
par le Dr JEAN BRISOU
}

Médecin de la Marine, doeteur ès sciences de l'Université de Bordeaux.

Il existe des lois, décrets et définitions codifiant la vente et la fabrication du beurre en France : articles I et III, loi du 16 avril $1897 ; 2^{e}$ Congrès international pour la répression des fraudes, Paris, du 17 au 29 octobre 1909 ; loi de 1897 , modifiée le 23 juillet 1907, reprise et complétée en 1929 et 1931 ; loi du 28 février et déeret du 30 octobre 1931.

Par contre, l'étude de la pureté bactérologique de eet aliment n'a pas encore franchi les portes du laboratoire.

En 1932, Dif́nert attire l'attention de l'Académie de Médecine sur la transmission possible de la typhoïde par le beurre. Après 